\title{
HOTSPOT STABILIZATION OF GOLD NANOPARTICLES FOR APPLICATION OF QUANTITATIVE SERS IN BIOANALYTICAL SYSTEMS
}

\author{
Mónica B. Mamián-López ${ }^{\mathrm{a}, \mathrm{b}, \#}$ and Marcia L. A. Temperini ${ }^{\mathrm{b}, *,(\bullet}$ \\ ${ }^{a}$ Centro de Ciências Naturais e Humanas, Universidade Federal do ABC, 09210-580 Santo André - SP, Brasil \\ bDepartamento de Química Fundamental, Instituto de Química, Universidade de São Paulo, 05513-970 São Paulo - SP, Brasil
}

Recebido em 27/05/2019; aceito em 28/08/2019; publicado na web em 21/10/2019

\begin{abstract}
A complete procedure including synthesizing gold nanoparticles and further hotspots stabilization is presented. Colloidal gold synthesis followed a citrate reducing reaction, then, an ideal condition for SERS enhancement with quantitative purposes was reached by searching equilibrium between salt- and analyte-induced aggregation. Under this condition, a mild aggregation is produced, along with "free sites" available on the metal surface where analyte could easily interact. As a consequence, suitable linear concentration intervals and high quality SERS spectra were obtained allowing building multivariate quantitative models. For adenine as probe molecule, LOD values were around $10^{-8} \mathrm{~mol} \mathrm{~L}^{-1}$, even at low laser power and short exposure time during spectral acquisition. The hotspot stabilization procedure, as shown here, allows dealing with samples or highly complex analytical matrices that can easily cause undesirable agglomerate formation like those from biological origin.
\end{abstract}

Keywords: SERS; gold nanoparticles; bioanalytical; adenine; quantitative SERS.

\section{INTRODUCTION}

Colloidal gold nanoparticles (AuNPs) have been attracting huge scientific interest for the past several years given their wide range of applications associate with its fascinating and unique chemical, optical and catalytic properties. As a key component of the surface enhanced Raman effect, observed for the first time around 45 years ago (1974), ${ }^{1,2}$ the fundamentals of growing metallic nanoparticles have been extensively studied and a variety of procedures for preparing them can be found in literature. ${ }^{3,4}$ When the conditions for generating the surface-enhanced effect are reached, the Raman signals for species with cross-sections as low as $10^{-30} \mathrm{~cm}^{-2}$ molecule ${ }^{-1}$ can be significantly intensified and, although in its first years, the SERS (Surfaceenhanced Raman Spectroscopy) technique was widely applied from a physical-chemist stand point, its remarkably increased sensitivity made it crossover to other branches of chemistry, especially analytical chemistry, where colloidal nanostructures are among the most popular substrate. Moreover, SERS analysis using AuNPs have become very popular in bioanalytical and biomedical applications, being used from single analyte detection in biological matrices, biomolecules sensing or drug-delivery system development and to more complex procedures such as carriers or intracellular imaging probes..$^{5-7}$

For quantitative purposes, one of the key steps is the colloid synthesis, a process that is still considered a mix of art and science due to the complexity of the growth and nucleation mechanisms behind it. ${ }^{8}$ Nowadays several experimental procedures are available; perhaps the most used methods are those described by Turkevich and Frens in $1951^{10,11}$ and the one reported by Lee-Meisel later in $1982 .{ }^{12}$ Briefly, both use sodium citrate, a mild reducing agent, added to a boiling aqueous solution of $\mathrm{HAuCl}_{4}$ and varying the concentration ratio between sodium citrate and gold (III) ion. These procedures supply red to violet colloidal suspensions of semi-spherical nanoparticles with size varying between 15 and $150 \mathrm{~nm}$ approximately. The Turkevich-Frens method has been carefully studied ${ }^{13}$ and different versions of this synthesis have been published. ${ }^{14}$ Although very

*e-mail: mlatempe@iq.usp.br

"e-mail alternativo: monikml@gmail.com low limits of detection (LOD) can be obtained, ${ }^{15}$ for quantitative purposes, ${ }^{16}$ SERS signal reproducibility is a requirement and it has been continuously pointed out since the first analytical applications arose. The reproducibility strongly depends on the way the hotspots (highly localized regions between AuNPs where enhancement occurs) are formed and on its efficiency in interacting with the analyte and on its stability. This condition requires equilibrium between stable agglomerates (usually generated using an external agent, i.e. $\mathrm{NaCl}$, $\mathrm{KCl}, \mathrm{MgSO} 4$, etc) and "free" hotspots to be filled with the analyte. When this condition is not adequately fixed, agglomerates can be too large and a competing aggregation process induced by the analyte itself (or components of a bioanalytical matrix) easily causes a colloid destabilization (a plasmon frequency red-shift). Until now, SERS reproducibility still remains as one of the major challenges to be overcome. ${ }^{17}$

Biological molecules have shown high affinity toward metallic nanostructures (mainly gold and silver) thus exhibiting spectra with high amounts of structural information but these advantages are easily lost when electrostatic stabilization (citrate in this case) of AuNPs is perturbed by competing phenomena. As a way to minimizing those extra contributors to the signal variability, this study is focused in an experimental approach for stabilizing hotspots while keeping a high SERS enhancement, reproducibility and sensitivity. We describe a modified Lee-Meisel colloid gold synthesis and a procedure for finding an ideal condition between the aggregation caused by a classical activating agent (i.e. a salt) and analyte-induced agglomerates, thus reaching a comfortable working condition simultaneously guaranteeing stable hotspots and enough "free" sites for analyte interaction. After that, a multivariate model suitable for analytical purposes will be build. For comparison, this procedure will be also applied on the classical Lee-Meisel colloid synthesis.

Here, we have chosen adenine as a main probe, due to its incontestable bioanalytical relevance, its well-known affinity for metallic nanoparticles as well as its fascinating and at the same time controversial orientation on metallic surfaces ${ }^{18}$ still being discussed in literature. Also, this experimental procedure has been tested in another adenine-related species, guanine, taking advantage of their notable differences in its affinities to AuNPs. ${ }^{19}$ The methodology 
exposed here, has been successfully applied in quantitative SERS $S^{20-22}$ analytical studies and has been optimized in continuous research in this field from more than six years now. ${ }^{23}$

\section{EXPERIMENTAL}

\section{Instrumentation}

Extinction spectra were acquired in a scanning spectrophotometer Shimadzu UVPC-3101. The SERS measurements were carried out using a Renishaw inVia confocal microscope system with a $20 \mathrm{X}$ long-working distance objective lens. As excitation source a 785 $\mathrm{nm}$ laser was used with power set to $5 \%$ of its maximum $(500 \mathrm{~mW})$. Scanning electron microscopy (SEM) images were taken with a JEOL Field Emission Gun model JSM-7401F, with an acceleration voltage of $5.0 \mathrm{kV}$. SEM images were analyzed using the software Image J (Version 1.52o 23, April 2019). Since AuNPs obtained are not entirely round, histograms were building using the largest dimension for each particle.

\section{Reagents and solutions}

All experiments were run in aqueous medium, using milli-Q water for all cases. Reagents were purchased from Sigma-Aldrich (Adenine $\left(\mathrm{C}_{5} \mathrm{H}_{5} \mathrm{~N}_{5}\right), 99.6 \%$; guanine $\left(\mathrm{C}_{5} \mathrm{H}_{5} \mathrm{~N}_{5} \mathrm{O}\right), 98 \%$; anhydrous sodium citrate, potassium chloride, chloroauric acid $\left(\mathrm{HAuCl}_{4}\right)$, $98 \mathrm{wt} \%$. Nitrogenous bases solutions were sonicated for about $15 \mathrm{~min}$ to improve solubilization.

\section{Gold Nanoparticles Synthesis}

Two syntheses of gold nanoparticles (AuNPs) have been carried out, the first one following the Lee-Meisel procedure and the second one, a modification of it, where $\mathrm{HAuCl}_{4}$ concentration was reduced to half its value. The complete experimental conditions for the syntheses are depicted in the Table 1 below.

Table 1. Experimental conditions for AuNPs synthesis

\begin{tabular}{lcc}
\hline \multirow{2}{*}{ Parameters } & \multicolumn{2}{c}{ Synthesis procedure conditions } \\
\cline { 2 - 3 } & Lee-Meisel & AuNPs-S2 $^{\mathrm{a}}$ \\
\hline $\mathrm{HAuCl}_{4}\left(\mathrm{mmol} \mathrm{L}^{-1}\right)$ & 1.2 & 0.50 \\
Sodium citrate $1 \%(\mathrm{~mL})$ & 10.0 & 3.0 \\
$\mathrm{n}_{\text {Sodium citrate }}$ to $\mathrm{n}_{\text {Au }}$ ratio & 3.3 & 2.4 \\
$\mathrm{~T}\left({ }^{\circ} \mathrm{C}\right)$ & b.p $\left(\sim 95^{\circ} \mathrm{C}\right)$ & b.p $\left(\sim 95^{\circ} \mathrm{C}\right)$ \\
\hline
\end{tabular}

aFrom now on, we will name the Au colloid solution obtained from the proposed synthesis in this work as AuNPs-S2.

\section{Hotspots stabilization}

Aggregation conditions for both colloids were adjusted searching a stable condition that would guarantee linearity in a range large enough to run an analytical quantification in absence of analyteinduced aggregation. This was accomplished in two stages:

First stage. Stabilization through an external activating agent

Successive additions of $\mathrm{KCl} 0.10 \mathrm{~mol} \mathrm{~L}^{-1}$ volumes $\left(\mathrm{V}_{\mathrm{i}}=50\right.$ to $500 \mu \mathrm{L})$ to AuNPs $(1.0 \mathrm{~mL})$ were made, monitoring their respective extinction spectra. Additions were stopped when a surface plasmon resonance (SPR) band drastically decreases and a new band (around $800 \mathrm{~nm}$ ) appeared. At this point, a first working range was chosen, containing 3 to 5 \{ AuNPs $\left.+\mathrm{V}_{\mathrm{i}}\right\}$ systems.
Second stage. Stabilization towards the analyte

After choosing $\left\{\right.$ AuNPs $\left.+V_{i}\right\}$ solutions, separate tests were made with each one of it by finding the minimum analyte concentration that would result in a SERS spectrum. The minimum detectable concentration was fixed when the most intense analyte's band appeared (either by visual inspection or when the signal-tonoise ratio reaches $\approx 3$ ). When the condition above was reached, the unique combination $\left\{\right.$ AuNPs $\left.+V_{i}\right\}$ chosen was the one where $V_{i}$ is now the minimum $\mathrm{KCl}$ volume allowing SERS signal.

As AuNPs spectrum didn't show any bands, their spectrum was considered as a blank in all cases. The volume ratio, AuNPs: $\mathrm{V}_{\text {analyte }}$, was either $10: 1$ or $5: 1$ as described previously. ${ }^{18}$

\section{Assessing a working range}

An estimation of a suitable working range was performed by increasing analyte concentration until the SPR band around $800 \mathrm{~nm}$ appears, indicating aggregation. The analyte concentration at this point was not considered for building the analytical model.

\section{Determination of hotspots stability through time}

The $\{$ AuNPs $+V i\}$ solution having analyte concentration for acquiring stability for successive SERS spectra measurements was evaluated using the analyte concentration where a SPR shifts in the colloids are observable, since at this point, the probability of suffering analyte-induced aggregation is higher. For each spectra sequence, $100 \mu \mathrm{L}$ of analyte solution were mixed with $1.0 \mathrm{~mL}$ of AuNPs, manually agitated and then it was transferred to a sample holder (usually Eppendorf cap). In the sequence, the sample was put under the microscope and the laser was focused on the colloid surface and this position at $z$ axis was set as zero. The laser focus was then displaced $200 \mu \mathrm{m}$ entering down in the sample. SERS spectra were acquired consecutively with single accumulations in different positions in the $x y$ plane. The procedure was performed for three replicates.

\section{Data processing}

Data processing was performed on Matlab 8.1.0.604 (R2013a). Baseline correction and multivariate approaches used for analytical modelling and performance were done using the PLS Toolbox (Eigenvector Research, Inc., version 8.5.2). For baselining procedure, a Weighted Least Squares (WLS) algorithm with a $2^{\text {nd }}$ order polynomial and smoothing using a Savitzky-Golay (5 points) filter were applied. PLS models were built with the entire spectra; the datasets were manually split into calibration and test samples, always test being the third part of the spectra.

\section{RESULTS AND DISCUSSIONS}

\section{AuNPs characterization}

Figure 1 presents the SEM images of AuNPs obtained from Lee-Meisel synthesis and that proposed in this work. Although both AuNPs have semi spherical morphology, the size dispersion for Lee-Meisel have shown to be relatively heterogeneous, ranging between 15 and $60 \mathrm{~nm}$ with its SPR located at $522 \mathrm{~nm}$. For AuNPs-S2 synthesis, a more homogeneous size distribution was observed with a mean size around $40 \mathrm{~nm}$, matching with its SPR at $532 \mathrm{~nm}$. 

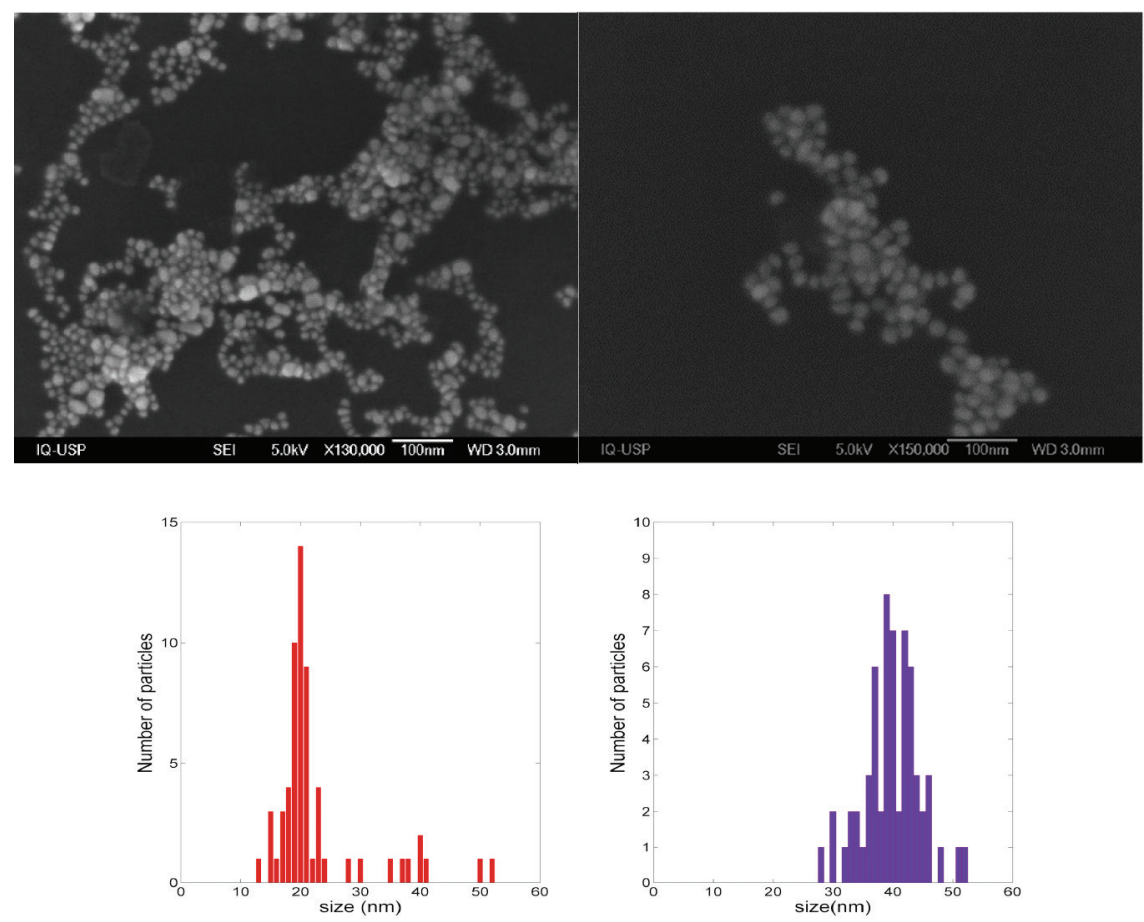

Figure 1. SEM images of AuNPs from of Lee-Meisel (left) and from AuNPs-S2 (right) syntheses and its respective dispersion size histograms

\section{Adenine as analyte}

\section{Hotspots stabilization}

As AuNPs aggregation is necessary to generate the highly localized enhancing regions, or hotspots, a careful procedure to reaching a working range to build an analytical curve was required. In a first step, successive additions of $\mathrm{KCl} 0.10 \mathrm{~mol} \mathrm{~L}^{-1}$ starting in 50 to $500 \mu \mathrm{L}$ were tested and the SPR was registered. As shown in Figure 2, a subtle SPR shift started to be observed when added volume was $250 \mu \mathrm{L}$. Upon strong aggregation $(\mathrm{V}=400 \mathrm{~mL})$, the intensity at $532 \mathrm{~nm}$ band is notably diminished and the extinction around $800 \mathrm{~nm}$ increased. At this point, a $\mathrm{KCl}$ volume selection between 50 and $250 \mu \mathrm{L}$ (five AuNPs activated) was done. Now, a new selection using adenine SERS spectra will be performed.

For the five $\{$ AuNPs $+\mathrm{Vi}\}$ solutions chosen, SERS spectra with adenine at $1.0 \times 10^{-4} \mathrm{mmol} \mathrm{L}^{-1}$ were tested but no signal was observed in this case. For the next upper concentration $\left(2.5 \times 10^{-4} \mathrm{mmol} \mathrm{L}^{-1}\right)$ tested, the band at $732 \mathrm{~cm}^{-1}$ (ring breathing vibration, py, $v$-ring) was observed for all five combinations previously chosen, thus, the one with the minimum $\mathrm{KCl}$ volume was fixed as working AuNPs system, $\left\{\right.$ AuNPs $+50 \mu \mathrm{L} \mathrm{KCl} 0.10 \mathrm{~mol} \mathrm{~L}^{-1}$ \}.

Although for the other four volumes the signal-to-noise ratio increases, it is recommended to stay at the minimum value (mild to low aggregation) guaranteeing thus that there is enough "free" sites in between the aggregates in a manner that if any analyte-induced aggregation is competing phenomenon still there would be possible to get a suitable enough linear signal-concentration range.

\section{Assessing a linear range}

The identification of a linear working range was carried out using SERS spectra for increasing adenine concentrations starting at $1.0 \times 10^{-4}$ until $0.10 \mathrm{mmol} \mathrm{L}^{-1}$. For illustration, the SPR spectra of Figure 3 were presented. Here, it is possible to observe and compare the analyte-induced aggregation phenomena for both

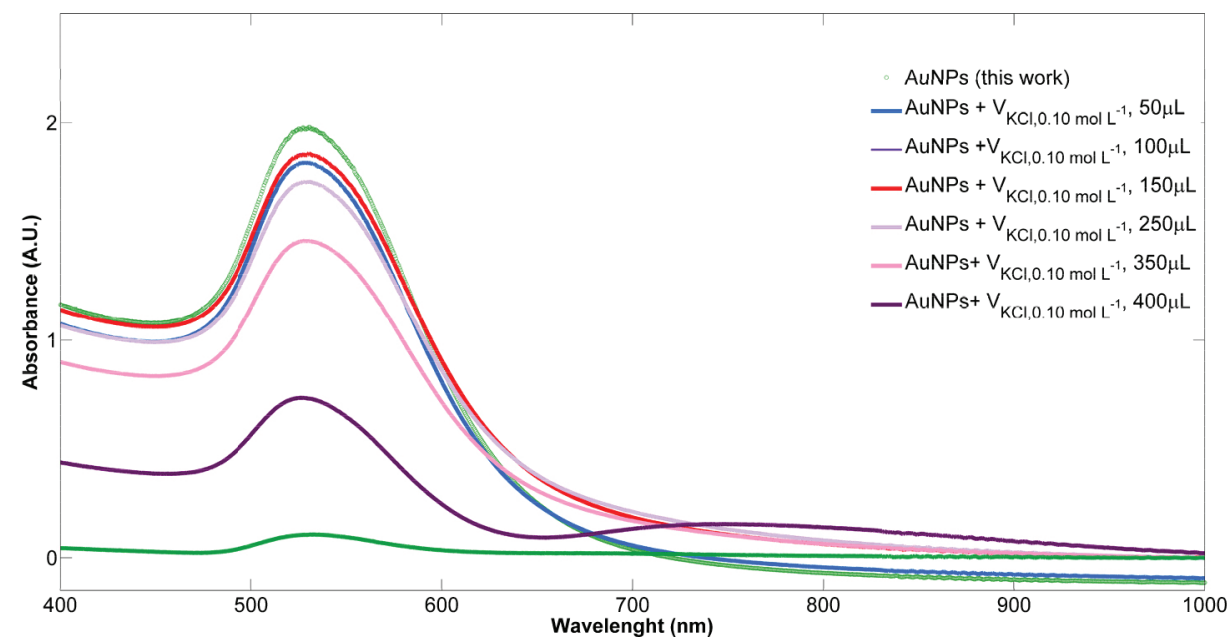

Figure 2. Extinction spectra of colloidal solution from AuNPs-S2 synthesis with addition of volumes of KCl $0.10 \mathrm{~mol} \mathrm{~L} \mathrm{~L}^{-1}$ between 50 and $400 \mu \mathrm{L}$. Classical activation procedure 
AuNPs synthesized. For AuNP-2 colloidal solution, the original SPR band $(533 \mathrm{~nm})$ is kept until adenine concentration get close to $0.05 \mathrm{mmol} \mathrm{L}^{-1}$ at which a new plasmon at about $800 \mathrm{~nm}$ starts to arise, indicating formation of bigger agglomerates and a higher probability of colloid destabilization induced by the analyte. A visual inspection of the extinction spectra allows concluding that below adenine 0.05 mmol L-1 there is a mild formation of aggregates, hence, the AuNPs are stable enough for SERS analysis and a linear range can be located between $1 \times 10^{-4}$ and $0.05 \mathrm{mmol} \mathrm{L}^{-1}$.

For the Lee-Meisel colloid, the scenario is quite different since in the evaluated concentration range, a rapid SPR shift is observed upon addition of adenine $0.005 \mathrm{~mol} \mathrm{~L}^{-1}$ and this behavior continues until almost disappearing of $520 \mathrm{~nm}$ band still at $0.05 \mathrm{mmol} \mathrm{L}^{-1}$.

\section{Hotspot stabilization through time}

One of the main worries when SERS reproducibility is questioned is the stability of a SERS signal through time in a real routine analysis. One source of instability is due to the fact that suspended particles (like in a colloid) will have a natural trend to create a concentration gradient from the top to the bottom of the sample cell. Instability in SERS spectra through the time also can originate from others very specific phenomena depending on the AuNPs-analyte system, for example when adsorption kinetics is slow enough changing the signal during the spectrum acquisition time.

For evaluation of the extent of the spectra stability in both colloids it was considered the acquisition time for a single spectrum (about $2 \mathrm{~min}$ ), with $10 \mathrm{~s}$ /accumulation. In order to observe the spectra behavior over time, the standard deviation (SD) for triplicate samples was calculated and, as depicted in Figure 4, a very noticeable dependence on the colloid used, was observed. For the Lee-Meisel colloid, SD reaches values as high as 50\% (normalized value) still at the beginning of the analysis, while for AuNPs-S2 colloid, values are around $12 \%$ and does not varies significantly until reaching the eighth acquisition (around 12-15 min since the first spectrum). It is clear that the stabilized AuNPs obtained following the procedure described in this work offers a comfortable working time, perfectly fitted to a routine analysis time (about 15 $\mathrm{min} / \mathrm{sample}$ ), with no significant changes in the final result, even for replicate measurements.

These observations are also accompanied by a big difference in the quality of the spectra (SERS enhancement, signal-to-noise ratio) along the work ranges used for both colloids. Just to illustrate this in the most "unstable" condition (the same at spectra were acquired for Figure 4), i.e., when agglomerates are already big, the adenine spectra shows a drastic difference in its SERS intensity, ten times higher for our synthesis, still considering that this enhancement could be improved by simply increasing the laser power, for example. It must be emphasized that adenine spectra obtained from AuNPs prepared following AuNPs-S2 synthesis, have a high signalto-noise ratio and bands are all clearly distinguishable, as shown in Figure 5. These features could come from a better or "cleaner" interaction between analyte and metal surface guaranteed by the
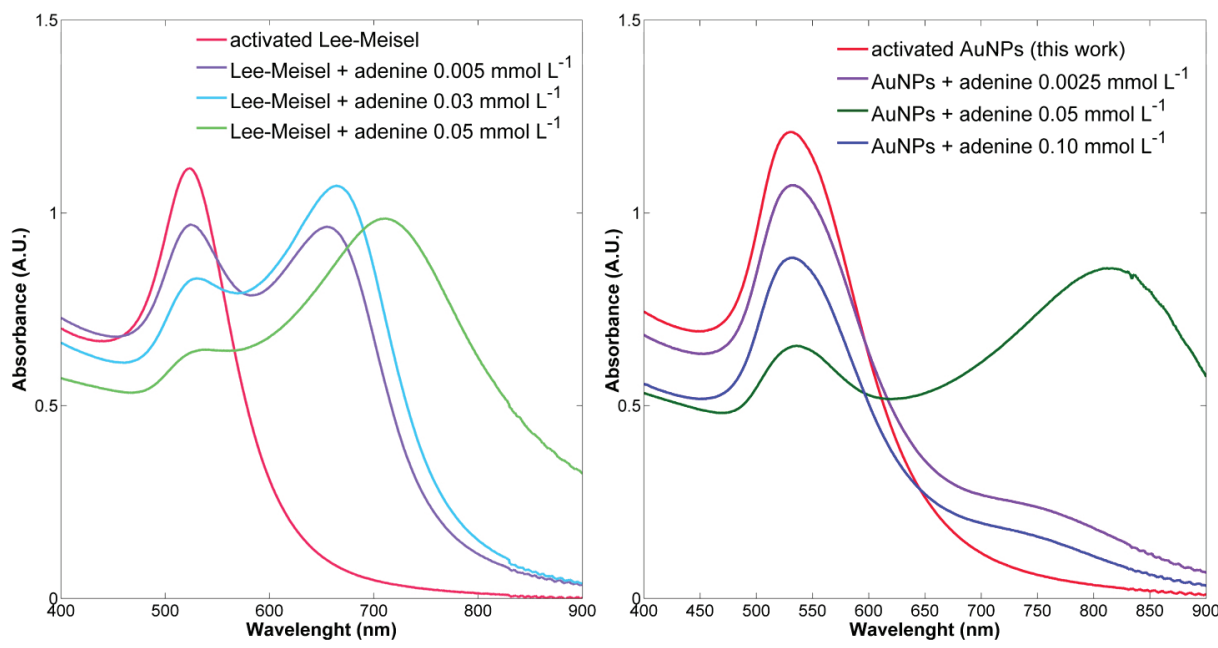

Figure 3. Extinction spectra of Au colloid from Lee-Meisel (left) and from AuNPs-S2 (right)syntheses, having adenine concentration between 0.005 and $0.10 \mathrm{mmol} \mathrm{L}^{-1}$
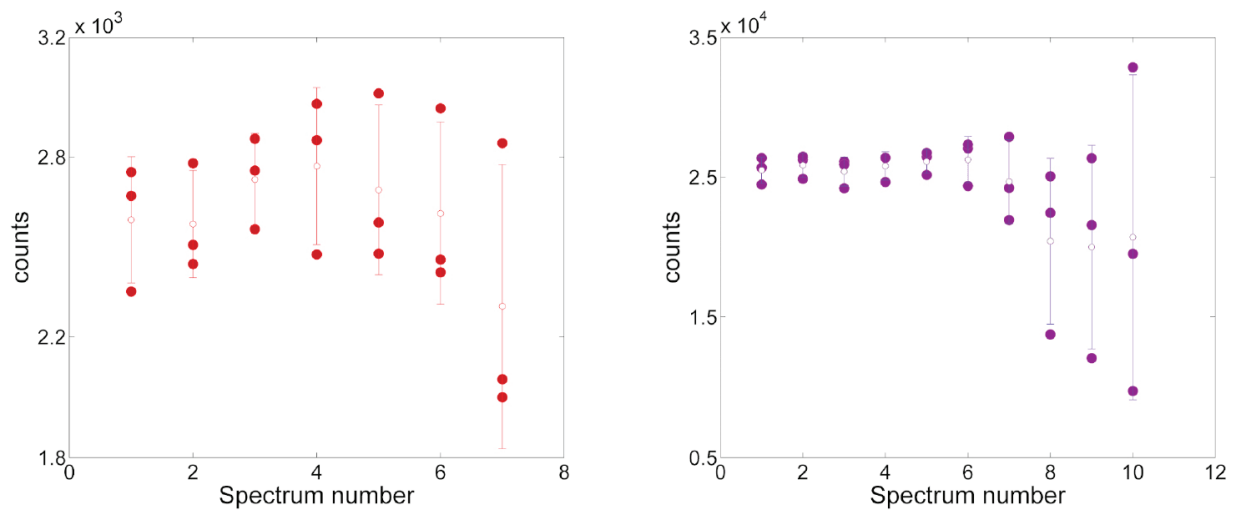

Figure 4. Intensity of adenine band at $732 \mathrm{~cm}^{-1}$ for successively acquired SERS spectra of three replicates using Au colloid from Lee-Meisel (left) and from AuNPs-S2 (right) syntheses. Bars indicates standard deviation to assess precision 

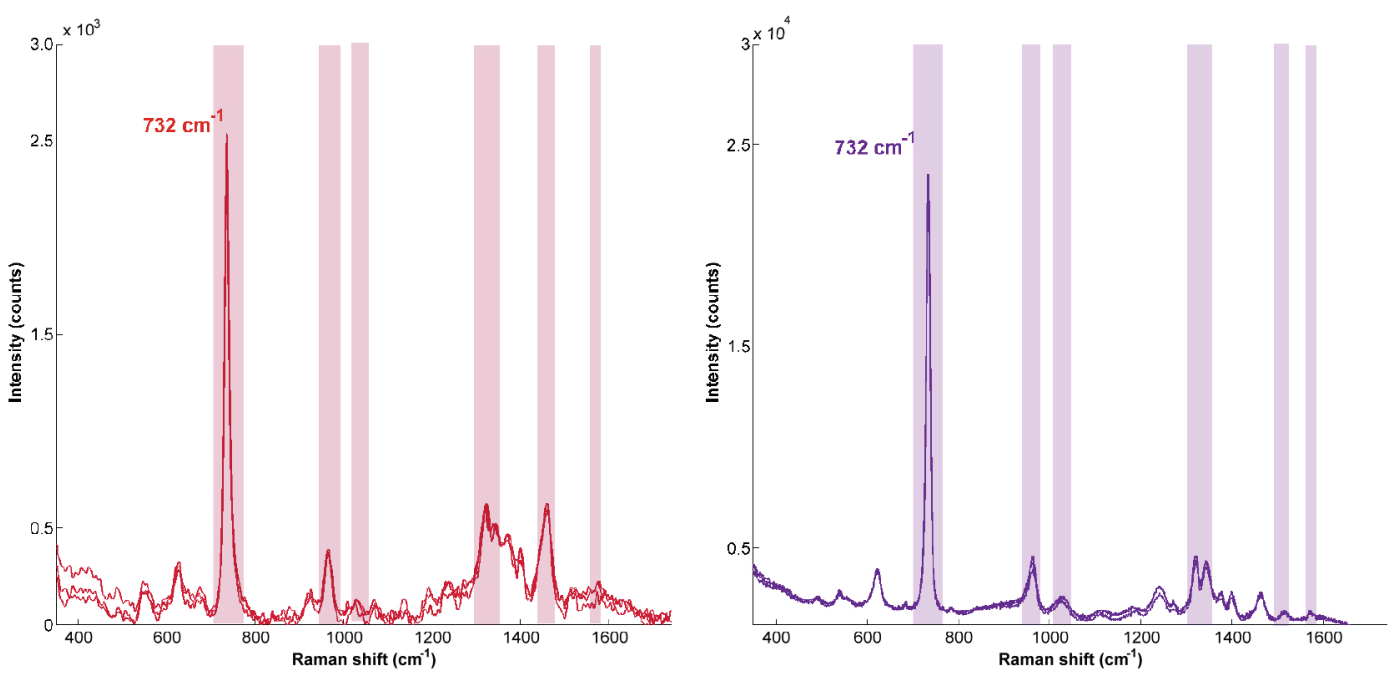

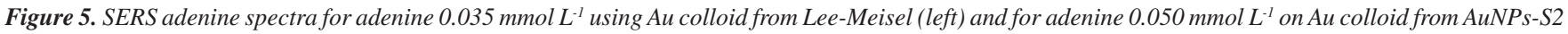
synthesis (right). Main bands highlighted located at $732 \mathrm{~cm}^{-1}, 960 \mathrm{~cm}^{-1}$ (from ring breathing and 5-ring deformation respectively), 1320 and $1350 \mathrm{~cm}^{-1}$ (from $-\mathrm{C}-\mathrm{N},-\mathrm{C}-\mathrm{C}-,-\mathrm{C}-\mathrm{H}-$, stretching and bending modes), $1026 \mathrm{~cm}^{-1}\left(\mathrm{NH}_{2}\right.$ rocking) , 1460 and $1550 \mathrm{~cm}^{-1}$ (in-plane $\mathrm{NH}_{2}$ scissoring)

mild aggregation reached. It is worth mentioning that the higher particle concentration in Lee-Meisel synthesis (two times higher than AuNP-S2), could eventually generate a comparable adenine SERS signal, but the probability of the spectra having higher noise contribution from spurious light scattering effects is significant. This is consistent with the observation in the extinction spectra of a strong SPR band from a new AuNPs agglomerates formed either induced by the $\mathrm{KCl}$ or analyte itself.

\section{Analytical performance}

For assessing the potential application of the procedure described in this work, where there is careful control of the analyteinduced aggregation, at first, a multivariate approach was applied. The complexity of biological matrices certainly will have many unidentified species with high probability of generating SERS signals turning necessary this kind of approach. One of the most popular methods is $\mathrm{PLS},{ }^{24}$ where multivariate models are built by reducing the data dimensionality to a few components (latent variables, LV) chosen accordingly parameters such as RMSECV (Root mean square error of cross-validation) and the percentage of explained variance accumulated (at least 90\%). The method allows using the entire spectra or intervals of it and one of its most attractive advantages is the ability to modeling, simultaneously with the analyte, unknown interferences without calibrating it (second-order advantage). ${ }^{25}$ After a calibration procedure, the resultant models should be able to predict the concentration (or the calibrated property) in new samples. The usefulness of this particular advantage becomes more remarkable when real samples are being analyzed since would be no need to know and hence to calibrate interferences coming from the analytical matrix. Even for first-order data as in our case, this advantage can be obtained.

Thus, two models (for Lee-Meisel colloid and AuNPs-S2) have been built, tested with new samples and its performance evaluated through the corresponding figures of merit. ${ }^{26,27} \mathrm{~A}$ first visual inspection of the models, depicted in Figure 6 (black and colored markers correspond to calibration and test samples, respectively), shows that the AuNP-S2 stabilized with the strategy described here allows a wider linear range without sacrificing spectra quality (see comparative Figure 5). It is also possible to roughly estimate the quality of precision, clearly better for the AuNPs-S2 model. The figures of merit allowed confirming those observations (see Table 2), LOD values are two orders of magnitude lower than for Lee-Meisel colloid, being

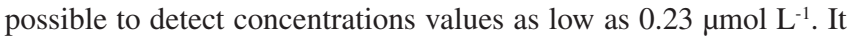
is important to consider that the spectra acquisition conditions used here (laser power, exposure time and number of accumulations) were low experimental set-up and this value could be potentially increased in further applications. Another useful indicative relies in the model calibration quality (expressed through it RMSECV value) and its predictive capability (RMSEP, root mean square error of prediction). Ideally, it is expected that a PLS model be able to perform a very good data modelling supplying a good prediction of new samples. The calculated values showed that the AuNP-S2 synthesis allows building a model with very similar predictive and modelling ability, while for Lee-Meisel colloid, despite a high linearity can be reached, predictions are significantly less accurate. Analytical sensitivity also matches with the fact that the procedure described in this work let to acquire spectra with high signal-to-noise ratio, guaranteeing detectable signal change in the order of $0.07 \mu \mathrm{mol} \mathrm{L}{ }^{-1}$, a noticeable difference with the value for Lee-Meisel synthesis, whose changes in SERS intensity are detectable with concentrations values around 50 times higher.

For comparison purposes, univariate curves using the adenine ring breathing band $\left(732 \mathrm{~cm}^{-1}\right)$ intensity are also included in Figure 6 (subplots). Here, differences are also evidenced, and although a linear behavior is possible with Lee-Meisel colloid, still the AuNP-S2 supplies a more fitted analytical curve. Additionally, a PLS model has been also built for the adenine analogous guanine using a different AUNPs-S2 batch, with very similar results (See Figures $1 \mathrm{~S}$ and 3S).

At this point we can conclude that, the synthesized AuNPs with further controlled aggregation as described here, should be suitable for applications including more complex systems such as biological fluids as already published, ${ }^{16}$ and even the high quality of obtained spectra has allowed using this conditioned AuNP-S2 to get a closer view to the interaction of DNA molecules as described previously. ${ }^{17}$ It is important to point out that the strategy presented here, should be easily adjusted to any system similar to our AuNPs-analyte, since the key stages for obtaining the desired results, relies mainly in the hotspot stabilization through both analyte and an external aggregating agent. If a stable condition far from extreme aggregation is set, there should be a suitable, comfortable working range for varying analyte concentration. 


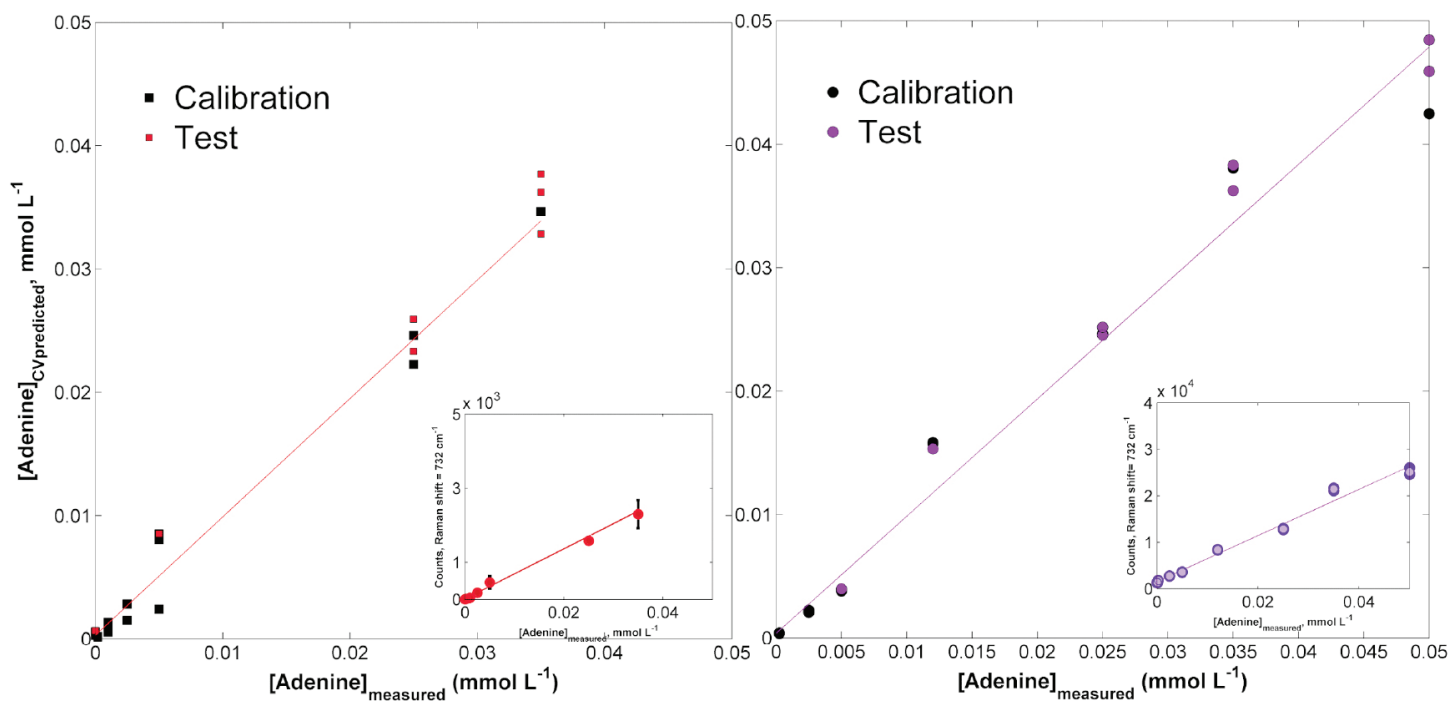

Figure 6. PLS models for adenine in concentrations ranging from 0.0 to $0.035 \mathrm{mmol} \mathrm{L} \mathrm{L}^{-1}$ on Lee-Meisel colloid (left) and from 0.0 to $0.05 \mathrm{mmol} \mathrm{L}^{-1} \mathrm{on}$ AuNP-S2 (right). CV predicted corresponds to predicted adenine concentrations calculated during cross-validation procedure

Table 2. Figures of merit for PLS models for adenine quantification using Lee-Meisel and AuNPs-S2 colloids

\begin{tabular}{|c|c|c|}
\hline \multirow{2}{*}{ Figures of merit } & \multicolumn{2}{|c|}{ PLS models } \\
\hline & Lee-Meisel & AuNPs-S2 \\
\hline RMSEC $^{\mathrm{a}}$ & 0.0002448 & 0.001590 \\
\hline $\mathrm{RMSECV}^{\mathrm{b}}$ & 0.001500 & 0.002220 \\
\hline RMSEP $^{c}$ & 0.001947 & 0.002211 \\
\hline $\mathrm{R}^{2} \mathrm{CV} / \mathrm{R}^{2} \mathrm{Cal}$ & $0.9796 / 0.9995$ & $0.9745 / 0.9865$ \\
\hline $\begin{array}{l}\text { \# LV / \%explained } \\
\text { variance by LV1 }\end{array}$ & $4 / 89.72$ & $2 / 94.5$ \\
\hline $\mathrm{LOD}\left(\mathrm{mmol} \mathrm{L}^{-1}\right)$ & 0.0142 & $2.25 \times 10^{-4}$ \\
\hline $\mathrm{LOQ}\left(\mathrm{mmol} \mathrm{L}^{-1}\right)$ & 0.0431 & $6.81 \times 10^{-4}$ \\
\hline $\begin{array}{l}\text { Analytical sensitivity } \\
\left(\mathrm{mmol} \mathrm{L}^{-1}\right)\end{array}$ & 0.0043 & $7.01 \times 10^{-5}$ \\
\hline
\end{tabular}

Root mean square error of a Calibration, ${ }^{\mathrm{b}} \mathrm{Cross}$-Validation, ${ }^{\mathrm{c} P r e d i c t i o n}$.

\section{CONCLUSIONS}

In this study, a detailed procedure including an adapted synthesis and focused in a careful hotspot stabilization process in gold nanoparticles was described. A comparison applying the same protocol for SERS as a quantitative technique was also performed using the classical Lee-Meisel colloid synthesis. The hotspots stabilization included controlling both salt- and analyte-induced aggregation and under these conditions a suitable working range and high-quality spectra could be obtained. It has been shown that the proposed procedure, applied on adenine as probe, let to drastically improve precision and accuracy in quantitative approaches, seen through the corresponding figures of merit (LOD, analytical sensitivity, RMSECV and RMSEP).

\section{SUPPLEMENTARY MATERIAL}

Supporting information containing SERS spectra for guanine with its respective PLS model, univariate curves for adenine, and additional SERS spectra for adenine are available in http://quimicanova.sbq. org.br, for free.

\section{ACKNOWLEDGMENTS}

The authors thank the FAPESP project 2016/21070-5. MLAT thanks to CNPq fellowship (302792/2015-5). MBML also thanks to CNPq fellowship (154066/2016-8). Authors are also thankful to G. T. Paganoto for the SEM images. Graphical abstract was a work by A. L. M. López.

References

1. de Faria, D. L. A.; Temperini, M. L. A.; Sala, O.; Quim. Nova 1999, 22, 541.

2. Fleischmann, M.; Hendra, P. J.; McQuillan, A. J.; Chem. Phys. Lett. 1974, 26, 163.

3. Mossier-Boss, P. A.; Nanomaterials 2017, 7, 142.

4. Qin, L.; Zeng, G.; Lai, C.; Huang, D.; Xu, P.; Zhang, C.; Cheng, M.; Liu, X.; Liu, S.; Li, B.; Yi, H.; Coord. Chem. Rev. 2018, 359, 1.

5. Goodacre, R.; Graham, D.; Faulds, K.; Trends Anal. Chem. 2018, 102, 359.

6. Jamieson, L. E.; Asiala, S. E.; Gracie, K.; Faulds, K.; Graham, D.; Annu. Rev. Anal. Chem. 2017, 10, 415.

7. Li, Y.; Wang, Z.; Mu, X.; Ma, A.; Guo, S.; Biotechnol. Adv. 2017, 35, 168.

8. Xia, J.; Xiong, Y.; Lim, B.; Skrabalak, S. E.; Angew. Chem., Int. Ed. 2009, 40, 60 .

9. Polte, J.; CrystEngComm 2015, 17, 6809.

10. Turkevich, J.; Hillier, J.; Stevenson, P. C.; Discuss. Faraday Soc. 1951, 11,55 .

11. Frens, G.; Nat. Phys. Sci. 1973, 241, 20.

12. Lee, P. C.; Meisel, D.; J. Phys. Chem. 1982, 86, 3391.

13. Kimling, J.; Maier, M.; Okenve, V.; Kotaidis, V.; Ballot, H.; Plech, A.; J. Phys. Chem. B 2006, 110, 15700.

14. Bastús, N.G.; Comenge, J.; Puntes, V.; Langmuir 2011, 27, 11098.

15. de Albuquerque, C. D. L.; Sobral Filho, R. G.; Poppi, R. J.; Brolo, A. G.; Anal. Chem. 2017, 90, 1248.

16. Kammer, E.; Olschewski, K.; Bocklitz, T.; Rosch, P.; Weber, K.; Cialla D.; Popp, J.; Phys. Chem. Chem. Phys. 2014, 17, 9056.

17. Goodacre, R.; Graham, D.; Faulds, K.; Trends Anal. Chem. 2018, 102, 359.

18. Harroun, S. G.; ChemPhysChem 2018, 19, 1003.

19. Barhoumi, A.; Zhang, D.; Tam, F.; Halas, N.; J. Am. Chem. Soc. 2008, 130, 5523. 
20. Mamián-López, M. B.; Corio, P.; Temperini, M. L.; Analyst 2016, 141, 3428.

21. Mamián-López, M. B.; Poppi, R. J.; Anal. Chim. Acta 2013, 760, 53.

22. Mamián-López, M. B.; Temperini, M. L.; Anal. Chem. 2018, 90, 14165.

23. Mamián-López, M. B.; Ph.D. Thesis, Universidade Estadual de Campinas, Brasil, 2013.
24. Lorber, A.; Kowalski, B. R.; Appl. Spectrosc. 1988, 42, 1572.

25. Booksh, K. S.; Kowalski, B. R.; Anal. Chem. 1994, 66, 782A.

26. Valderrama, P.; Braga, J.; Poppi, R. J.; Quim. Nova 2009, 32, 1278.

27. Sena, M. M.; Chaudhry, Z. F.; Collins, C. H.; Poppi, R. J.; J. Pharm. Biomed. 2004, 36, 743.

FAPESP helped in meeting the publication costs of the article 